\title{
A RARE CASE OF CONGENITAL LEFT VENTRICULAR ANEURYSM WITH MITRAL REGURGITATION AND ABSENT LOWER LIMB PULSATIONS
}

\author{
Anand L. Betdur1', Vijaysai V. C², Raja Ponnambalam³, Somsekar D. S4, Naveen Kumar P5
}

\section{HOW TO CITE THIS ARTICLE:}

Anand L. Betdur, Vijaysai V. C, Raja Ponnambalam, Somsekar D. S, Naveen Kumar P. "A Rare Case of Congenital Left Ventricular Aneurysm with Mitral Regurgitation and Absent Lower Limb Pulsations". Journal of Evolution of Medical and Dental Sciences 2014; Vol. 3, Issue 18, May 05; Page: 4881-4885,

DOI: $10.14260 /$ jemds/2014/2528

ABSTRACT: Left ventricular aneurysms are not uncommon and mostly occur as a sequel of acute myocardial infarction. Congenital left ventricular aneurysms (CLVA) especially submitral ones are very rare and only few cases are reported in literature. Here we report a unique case of congenital sub mitral aneurysm, in a 16 year old patient presenting as mitral regurgitation with absent lower limb pulsations.

KEYWORDS: Left ventricular aneurysm, Mitral regurgitation, absent pulsations.

INTRODUCTION: Left ventricular aneurysms can be congenital or acquired. They develop most frequently after myocardial infarction. Other causes include Hypertrophic cardiomyopathy, Arrythmogenic right ventricular cardiomyopathy, myocarditis, chest trauma, sarcoidosis, Chaga's disease and without any identifiable cause. ${ }^{1}$ A congenital left ventricular aneurysm is a rare condition involving protrusion or out pouching of a portion of the ventricular wall with a broad connection to the ventricular cavity which may or may not have paradoxical systolic expansion. ${ }^{2}$ The combination of CLVA with mitral insufficiency is rare. The largest series of these kinds of aneurysms that were found in the literature is 6 cases over a period of 11years, described by Elio Marijon et al in 2006. ${ }^{3}$

We report one such rare and unique case of CLVA with mitral regurgitation and absent lower limb pulsations.

CASE REPORT: A 16 year old male patient admitted in the department of medicine, of our hospital on $18^{\text {th }}$ July 2013 was apparently all right 2 years back, when he developed insidious on set pain in right calf muscles since 18 months which was increasing on walking and subsiding on taking rest.

One month before admission he developed left sided catchy type of chest pain which was increasing on exertion and relieved on taking rest. He also had progressive exertional dyspnea (NYHA Grade I-III). He did not give any history of Paroxysmal nocturnal dyspnea, orthopnea. There was no history of palpitation, syncope, giddiness. There was no h/o cough, fever and constitutional symptoms were not present.

No h/o alcohol intake, smoking or tobacco consumption.

There was no past history of chest pain, dyspnea, palpitation associated with fever. No past history of chronic cough, joint pains, skin lesions. No past h/o exposure to sexually transmitted disease.

No past history of trauma to the chest.

No family history of premature deaths or history suggestive of cardiac disease.

ON EXAMINATION: He was moderately built and moderately nourished. He had no pallor, icterus, cyanosis, pedal edema and was afebrile. His right radial pulse was $84 / \mathrm{min}$, irregular, varying in 


\section{CASE REPORT}

volume and character. Popliteal, dorsalis pedis, posterior tibial were not palpable bilaterally. His blood pressure was 120/84 mm of $\mathrm{Hg}$ in right arm. Supine position and JVP was not raised.

Examination of cardiovascular system revealed prominent $S_{3}$ and a high pitched pan systolic murmur of grade $3 / 6$ heard at the apex, radiating to axilla. $\mathrm{P}_{2}$ was loud.

Respiratory system and per abdominal examination were normal. No neurological deficit was present.

On investigating, the patient had normal complete blood counts. ASLO titers were 61.9IU/lit, CRP $1.87 \mathrm{mg} / \mathrm{dl}$, VDRL.HIV, HBs Ag, Hepatitis C antibody were negative. INR 1.34 with Ratio 1.33\%. Prothrombin time $14.0 \mathrm{sec}$. Serum electrolytes were normal.

The patient's ECG-showed heart rate of 102 /min, prolonged PR interval, ST depression in II.III, aVF, $\mathrm{V}_{3}$ to $\mathrm{V}_{6}$. and borderline LVH. Chest $\mathrm{x}$ - showed increased cardio thoracic ratio of 0.6 with normal lung fields.

Holter monitoring showed prolonged PR interval, Mobitz type II heart block and sinus pauses.

Trans thoracic echocardiography (TTE) revealed-large LV Aneurysm of sub mitral type. Below and posterior to aortic valve, of size $52 \times 33 \mathrm{~mm}$, communicating with LV cavity (Fig. 1). Anterior mitral leaflet was distorted due to the aneurysm. Moderate eccentric mitral regurgitant jet was seen, directed posteriorly (Fig. 2). Left atrium, left ventricle mildly dilated. Good left ventricular and right ventricular function. No clot/vegetation. Aorta and arch were normal.

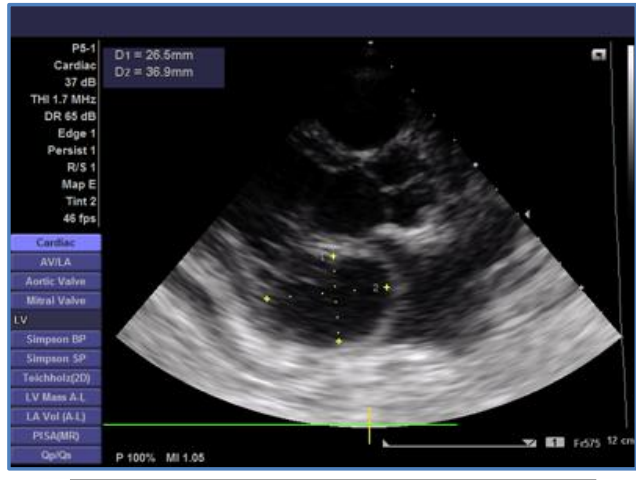

Fig. 1: TTE Showing Large Sub Mitral LV Anesrysm

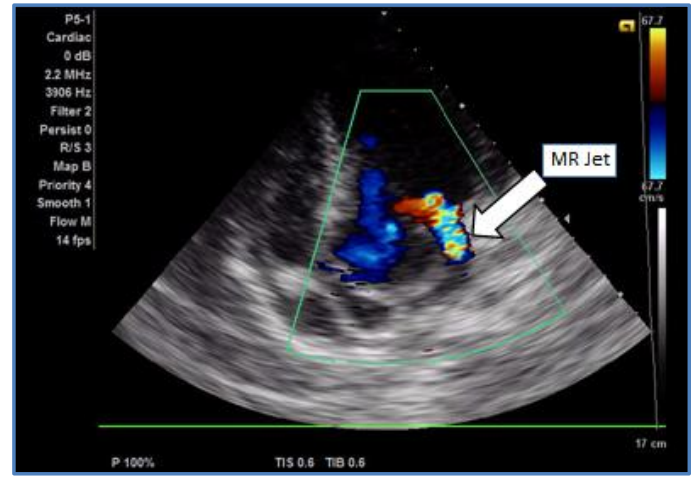

Fig. 2: TTE Showing Eccentric MR Jet

Patient was subjected to trans esophageal echocardiography (TEE) which confirmed large sub mitral LV aneurysm (Fig. 3).

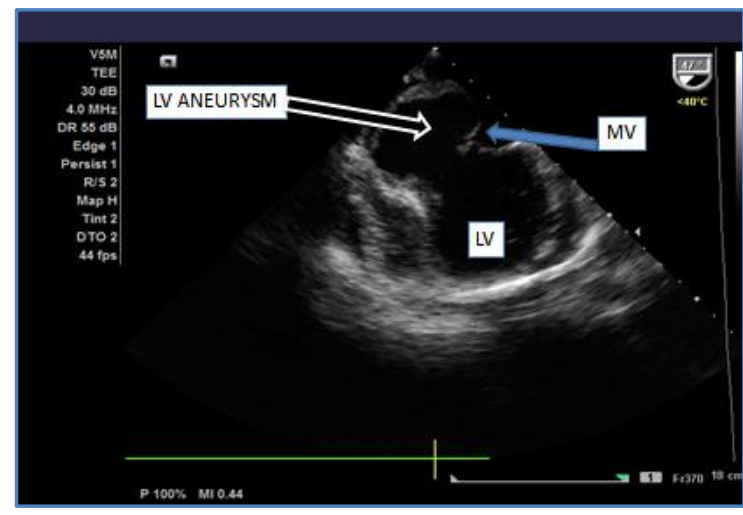

Fig. 3: TEE Showing Large Sub Mitral LVA 
Patient underwent coronary angiogram (CAG), left ventricular and peripheral arterial angiogram (PAG) via right brachial artery as a part of evaluation. CAG revealed normal epicardial coronaries, aorta and sub clavian arteries. LV angiogramconfirmed large sub mitral aneurysm communicating with LV cavity. (fig. 4).

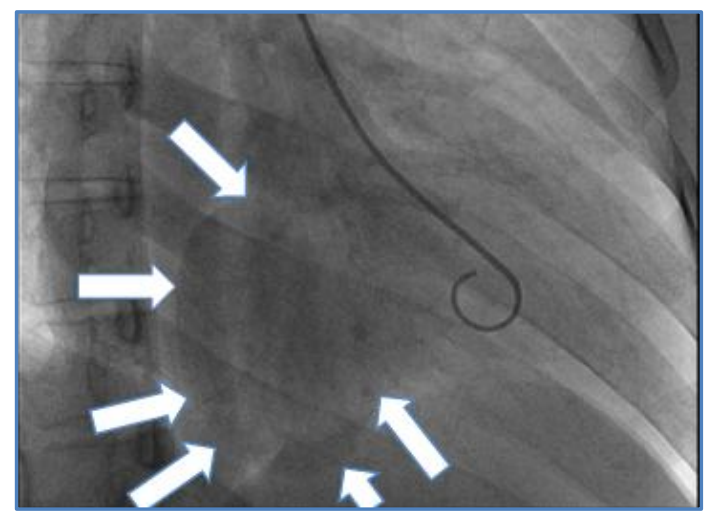

Fig. 4: LV Showing Large Sub Mitral Aneurysm Communicating With Cavity

PAG revealed normal iliac vessels. Renal arteries normal.20-25\% lesion at right common femoral artery. Both superficial femoral arteries showed long segment lesions in mid-thigh region. Reformation of both popliteal arteries seen by collaterals (fig. 5). Both anterior tibial arteries are not visualized; posterior tibial arteries are under filled.

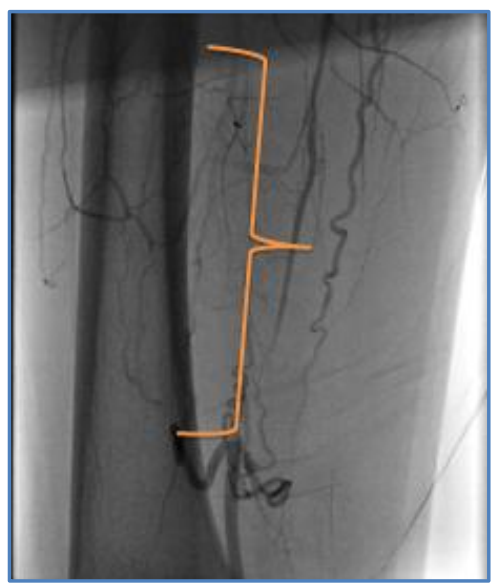

\section{Fig. 5: PAG Showing Segment Block in Superficial Femoral Artery}

With findings of normal coronaries in a young patient and after excluding infective and other acquired causes of LVA clinically and by ECHO, TEE. and CAG, we made a diagnosis of CONGENITAL, SUB MITRAL, LEFT VENTRICULAR ANUERYSM WITH MITRAL REGURGITATION.

Patient was put on T. Ecosprin 150mg 0-1-0.Acitrom 2mg o-o-1 and was advised to undergo aneuresmectomy at the earliest.

DISCUSSION: Most cardiac LVAs are acquired due to coronary artery disease \& myocardial infarction (MI) in adult hood. Uncommonly other causes of LVA are reported. LVAs could be of cardiac and non cardiac etiology. Cardiac causes apart from MI are coronary artery fistula, Hypertrophic obstructive 


\section{CASE REPORT}

cardiomyopathy, Arrythmogenic Right ventricular cardiomyopathy, and myocarditis. Non-cardiac causes include sarcoidosis, Chagas disease, Systemic lupus erythematosus, Behcet's disease, tuberculosis, HIV, Syphilis and Glycogen storage disease and blunt chest trauma ${ }^{4}$

A congenital LVA is a rare cardiac manifestation, a study reported an incidence of $0.4 \%$ of 750 cardiac necropsies. ${ }^{5} 411$ cases have been reported since its first description in $1816 .{ }^{6}$ Elio Marjoli et al reported 6 cases over a period of 11 yrs. Very few cases of congenital sub mitral LVAs are reported in literature. ${ }^{3}$ Our case of congenital sub mitral LVA is unique in presentation with claudication pain due to long segment blocks in both superficial femoral vessels. This could be due to multiple silent thrombo embolic episodes, however our case did not show any LV clot.

LV aneurysms must be distinguished from Left ventricular diverticula. The walls of a diverticulum are formed by all three layers and contracts normally. whereas an aneurysm is generally a fibrous saccular region with paradoxical contraction. ${ }^{7}$ The true incidence of sub-valvar aneurysms is certainly underestimated, especially in the early stages when there are no clinical symptoms. The etiology of sub valvular aneurysm is mostly thought to be a congenital weakness of the fibrous annulus of the valve, which is further supported by the predominantly race group as well as anatomy and embryology of the affected area. ${ }^{8}$

Most LVAs are asymptomatic and are occasionally identified during routine diagnostic procedures. Sudden cardiac death could be the first manifestation. They may present with systemic embolism, valve regurgitation, ventricular rupture, or ventricular arrhythmias, sometimes life threatening. ${ }^{2}$

Our patient did not have clinical features of arrhythmias, but ECG showed conduction disturbances.

Surgical repair is the gold standard; however controversy exists about surgical approach and technique. ${ }^{9}$ In view of their high rate of complications some authors advocate surgical resection even if they are asymptomatic. ICD and Radio frequency catheter ablation have been used successfully for the treatment of VT7. Prognosis of LVAs depends upon size, location, degree of valve involvement, functional class and presence of arrhythmias.

CONCLUSION: Congenital left ventricular aneurysms though rare, should be considered in any young patient with unexplained symptoms of heart failure. They can cause life threatening complications like ventricular tachycardia and sudden death, which are preventable by surgery, intra cardiac defibrillator, or radio ablation. CLVAs can be easily picked up by transthoracic echocardiography.

\section{REFERENCES:}

1. Stelios Paraskevaidis, George Stavropoulos, Vassilios Vassilkos, Yiannis S. Chatzizisis, Kostas Polymeropoulos, Anthony Ziakas, et al. Idiopathic Left ventricular aneurysm causing ventricular tachycardia with 1:1 ventriculoatrial conduction and intermittent Wenckebach block. The open cardiovascular Medicine Journal 2009; 3: 105-109.

2. Nitin Chaubal, Manjiri Dighe, Mohit Shah, Jyothi Chaubal, Jayanti Raghavan. Congenital left ventricular Aneurysm. J Ultrasound Med2004; 23: 125-128.

3. Michel Francklyn Mitsomoy, Mohomed Reda Ajaja, Btisam Fkiri, L Haddour, Younes Cheikhaoui. Management of the congenital aneurysm of the left ventricle Associated with Mitral Insufficiency in a child. Journal of Cardiovascular and Thoracic Research 2013; 5(1):35-36. 
4. Mattias Paul, Michael Scafers, Matthias Grude, Florian Reinke, Kai UweJuergens, Roman Fischbach, et al. Idiopathic left ventricular aneurysm and sudden cardiac death in young adults Europace 2006;8:607-612.

5. Ruth Perez-Fernandez, Rodrigo Medina-Alba, Ramon Mantilla, Rafeela Soler, Gonzalo Pradas, and Manuel Penas Lado. Congenital apical left ventricular aneurysm. Rev Esp Cardiol. 2005; 58(11):1361-3.

6. Ohlow M A. Congenital left ventricular aneurysm and diverticula: definition, pathophysiology. clinical relevance and treatment. Cardiology 2006; 106 (2):63-72.

7. Satoshi Yamashiro, Yukio Kuniyoshi, Kazufumi Miyagi, Toru Uezu, Katsuya Arakaki, Kageharu Koja. Two cases of ventricular tachycardia with congenital left ventricular malformation in an adult. Ann Thorac Cardiovasc Surg 2004; 10:42-46.

8. Henning J. Du Toit, Ulrich O. Von Oppell, John Hewitson, John Lawrenson, John Davies. Left ventricular sub-valvar mitral aneurysms. Interactive Cardiovascular and Thoracic Surgery 2003; 2: 547-551.

9. Sudarshan G T, Kanak N Nagle, Kamlesh Jain, Bipeen Y Bhamre, Athouba Arambam Singh, Tushar G Dhopade. A Rare case of submitral Left ventricular aneurysm with mitral Regurgitation. Bombay Hospital Journal 2011; 53, No.1:120-23.

\section{AUTHORS:}

1. Anand L. Betdur

2. Vijaysai V. C.

3. Raja Ponnambalam

4. Somsekar D. S.

5. Naveen Kumar P.

\section{PARTICULARS OF CONTRIBUTORS:}

1. Associate Professor, Department of Medicine, Vydehi Institute of Medical Sciences and Research Centre, Whitefield, Bangalore.

2. Associate Professor, Department of Cardiology, Vydehi Institute of Medical Sciences and Research Centre, Whitefield, Bangalore.

3. Professor, Department of Medicine, Vydehi Institute of Medical Sciences and Research Centre, Whitefield, Bangalore.
4. Professor and HOD, Department of Medicine, Vydehi Institute of Medical Sciences and Research Centre, Whitefield, Bangalore.

5. Post Graduate Student, Department of Medicine, Vydehi Institute of Medical Sciences and Research Centre, Whitefield, Bangalore.

\section{NAME ADDRESS EMAIL ID OF THE CORRESPONDING AUTHOR:}

Dr. Anand L. Betdur, Associate Professor, Department of Medicine, Vydehi Institute of Medical Sciences and Research Centre, Whitefield, Bangalore.

E-mail: dranandbetdur@yahoo.co.in

Date of Submission: 08/04/2014. Date of Peer Review: 09/04/2014. Date of Acceptance: 21/03/2014. Date of Publishing: 03/05/2014. 